\title{
WECKEN THEOREMS FOR NIELSEN INTERSECTION THEORY
}

\author{
CHRISTOPHER K. McCORD \\ Institute for Dynamics, Department of Mathematics, University of Cincinnati \\ P.O. Box 210025, Cincinnati, Ohio 45221-0025, U.S.A. \\ E-mail: chris.mccord@uc.edu
}

\begin{abstract}
Nielsen theory, originally developed as a homotopy-theoretic approach to fixed point theory, has been translated and extended to various other problems, such as the study of periodic points, coincidence points and roots. Recently, the techniques of Nielsen theory have been applied to the study of intersections of maps. A Nielsen-type number, the Nielsen intersection number $N I(f, g)$, was introduced, and shown to have many of the properties analogous to those of the Nielsen fixed point number. In particular, it is a homotopy-invariant lower bound for the number of intersections of a pair of maps. The question of whether or not this lower bound is sharp can be thought of as the Wecken problem for intersection theory. In this paper, the Wecken problem for intersections is considered, and some Wecken theorems are proved.
\end{abstract}

1. Introduction. Nielsen fixed point theory, a homotopy-theoretic approach to fixedpoint theory, grew out of Nielsen's work in the 1920's on surface homeomorphisms. From those origins, Nielsen fixed point theory has grown into a richly developed theory. Moreover, the methods of Nielsen theory have been translated from fixed point problems into other domains, such as the study of periodic points, coincidence points, roots, \&c. That is, there are Nielsen coincidence numbers, Nielsen root numbers, \&c., defined in the similar fashion, and with similar properties, as the original Nielsen fixed point number. In [10], the Nielsen machinery was adapted into another new setting: intersections of maps. In that paper, a wide variety of results for Nielsen intersection numbers were presented. In this paper, we restrict ourselves to examining the definition of the Nielsen intersection number and the Wecken property for intersection numbers. We will see that, even in these basic issues, there are some interesting subtleties and unresolved questions.

1991 Mathematics Subject Classification: Primary 55M20.

Supported in part by grants from the National Science Foundation and the Taft Foundation. The paper is in final form and no version of it will be published elsewhere. 
The data for the intersection problem consists of a triple of spaces $X, Y, Z$, and a pair of maps $f: X \rightarrow Z$ and $g: Y \rightarrow Z$. Given this data, the intersection set of $f$ and $g$ is

$$
\operatorname{Int}(f, g)=\{(x, y) \in X \times Y \mid f(x)=g(y)\} .
$$

In general, the intersection set can be quite complicated, and the problem of "describing $\operatorname{Int}(f, g)$ " correspondingly intractible. In settings such as algebraic geometry, there are highly developed theories which are tailored to that setting. For a Nielsen theory (i.e. an algebraic-topological theory), two categories are appropriate: a topological category, in which all of the spaces involved are compact, Hausdorff, path-connected and admit universal covering spaces; and the subcategory of smooth manifolds. In either setting, the intersection set is clearly compact.

Actually, it is not exactly "describing $I(f, g)$ " for a single pair of maps $f$ and $g$ that we are interested in. If either $f$ or $g$ is deformed by a homotopy, the intersection set will change. In particular, we can create arbitrarily large intersection sets via homotopies of $f$ and $g$. To discount the contribution of such spurious intersection points, we will instead describe the minimal intersection set. There are two alternatives to consider here: counting points in the intersection set; or counting path components in the intersection set. That is, we can define

$$
M I_{p}(f, g)=\min \left\{\left|I\left(f^{\prime}, g^{\prime}\right)\right| \mid f^{\prime} \simeq f, g^{\prime} \simeq g\right\}
$$

and

$$
M I_{c}(f, g)=\min \left\{\left|\pi_{0}\left(I\left(f^{\prime}, g^{\prime}\right)\right)\right| \mid f^{\prime} \simeq f, g^{\prime} \simeq g\right\} .
$$

Deferring to $\S 4$ the discussion of the relative merits of the two definitions, we content ourselves for now with the trivial observation that $M I_{c}(f, g) \leq M I_{p}(f, g)$.

Our goal is to develop a Nielsen intersection number $N I(f, g)$ that will provide a lower bound for $M I_{c}(f, g)$. We briefly sketch the definition of $N I(f, g)$ here, and give a more complete presentation in $\S 3$. As with any "Nielsen-type number," the first step is to partition the intersection set $\operatorname{Int}(f, g)$ into equivalence classes. The equivalence relation is chosen to make precise the intuitive idea that two intersection points should be equivalent if one can be continuously deformed into the other. In particular, every equivalence class is a union of path components, and hence isolated. Once the intersection set has been partitioned, we need a criterion to decide which intersection classes are essential, and which are inessential. Here again, two alternatives present themselves. When an intersection index can be defined, we can declare an intersection class to be algebraically essential if it has non-zero index, and define the algebraic Nielsen number $N I_{a}(f, g)$ to be the number of algebraically essential intersection classes. But this is one of the crucial differences between the fixed point problem on the one hand, and the coincidence, root and intersection problems on the other. A fixed point index can be defined in great generality; while the other problems only admit useful algebraic indices in special circumstances. Roughly speaking, in all three problems, all of the spaces must be compact orientable manifolds with restrictions on the dimensions ${ }^{1}$. The definition of

\footnotetext{
${ }^{1}$ Dobreńko and Jezierski have developed a semi-index which can be applied to the coincidence problem on non-orientable manifolds [5]; and Kucharski and Eęcki are extending this to the (generalized) root problem on non-orientable manifolds.
} 
the intersection index for compact orientable manifolds with $\operatorname{dim}(X)+\operatorname{dim}(Y)=\operatorname{dim}(Z)$ is briefly reviewed in $\S 2$.

Since the intersection index is only defined in a limited, albeit important, setting, we need a more general concept of an essential intersection class. The same difficulty is encountered in the coincidence and root problems, and we use the techniques employed by Brooks [1, 2] and Dobreńko and Kucharski [4] in those problems. That is, we declare a class to be topologically inessential if some homotopy of $f$ and $g$ can remove the class, and topologically essential otherwise. The number of topologically essential intersection classes gives us the Nielsen intersection number $N I(f, g)$. Now, the whole point of introducing the intersection index is that a class with non-zero index cannot be removed by homotopy. That is, when the index is defined, algebraically essential classes are topologcially essential, and $N I_{a}(f, g) \leq N I(f, g)$. It is also clear that $N I(f, g) \leq M I_{c}(f, g)$. Thus we have four quantities of interest, related by the inequalities

$$
N I_{a}(f, g) \leq N I(f, g) \leq M I_{c}(f, g) \leq M I_{p}(f, g) .
$$

It is natural to ask if these inequalities are ever equalities. Questions of this type can be thought of as the Wecken problem for intersection theory. I tentatively suggest the following terminology: maps $f$ and $g$ satisfy the weak Wecken property if the equality $N I(f, g)=M I_{c}(f, g)$ holds; and the strong Wecken property if the equalities

$$
N I_{a}(f, g)=N I(f, g)=M I_{c}(f, g)=M I_{p}(f, g)
$$

hold. Similarly, the triple of spaces $X, Y, Z$ satisfy the strong or weak Wecken property if the appropriate equalities hold for all $f: X \rightarrow Z \leftarrow Y: g$.

The core of this paper, $\S \S 4$ and 5 , is devoted to the Wecken problem for intersection numbers. The main result, analogous to [3, Theorem 2] for coincidences and [4, Theorem 3.4] for roots, is that the strong Wecken property holds for manifolds, with a few low dimensional exceptions. Namely,

ThEOREM 1.1 ([10, Theorem 4.4]). If $X, Y$ and $Z$ are compact orientable manifolds such that $\operatorname{dim}(Z) \geq \operatorname{dim}(X)+\operatorname{dim}(Y)$ and $\max \{\operatorname{dim}(X), \operatorname{dim}(Y)\}>2$, then the triple $(X, Y, Z)$ satisfies the strong Wecken property: for every $f: X \rightarrow Z$ and $g: Y \rightarrow Z$,

$$
N I_{a}(f, g)=N I(f, g)=M I_{c}(f, g)=M I_{p}(f, g) .
$$

In particular, there exists $f^{\prime} \simeq f$ and $g^{\prime} \simeq g$ such that $f^{\prime}$ and $g^{\prime}$ have exactly $N I(f, g)$ points of intersection, each of which is algebraically essential.

We will also see in $\S 5$ that the strong Wecken property also holds in some low dimensional situations.

To the reader familiar with $[3,4]$, the author's intellectual debt to Brooks and Dobreńko and Kucharski is clear. Much of this work is simply an application of their methods in a new setting. Beyond the obvious stylistic similarities, the three problems - the coincidence problem considered by Brooks, the "generalized" problem or root problem considered by Dobreńko and Kucharski and the intersection problem considered by the author - exhibit a deeper structural relation. While formally distinct, any of the three problems can in a precise and formal fashion be transformed into either of the other 
problems. This suggests that the three Nielsen theories should be closely related. In a separate work [11], the author will explore these relationships.

2. The intersection index. Suppose that $X, Y$ and $Z$ are compact, orientable manifolds of dimensions $p, q$ and $n=p+q$ respectively. Fix orientations for $X, Y$ and $Z$. A set $J \subset \operatorname{Int}(f, g)$ is isolated set of intersections if there exists a neighborhood $U \subset X \times Y$ of $J$ such that $\operatorname{Int}(f, g) \cap U=J$. In this setting, there are two equivalent ways to define the intersection index (cf. [7]).

On the one hand, we can, by an arbitrarily small perturbation of $f$ and $g$, assume that the maps are smooth and transverse at every point of intersection. For every $(x, y) \in J$, take a basis $\left\{v_{1}, \ldots, v_{p}\right\}$ for $T_{x} X$ and a basis $\left\{w_{1}, \ldots, w_{q}\right\}$ for $T_{y} Y$. Then

$$
\left\{D f_{x}\left(v_{1}\right), \ldots, D f_{x}\left(v_{p}\right), D g_{y}\left(w_{1}\right), \ldots, D g_{y}\left(w_{q}\right)\right\}
$$

forms a basis for $T_{f(x)} Z$. If this basis has the same orientation as that fixed for $Z$, we define $\operatorname{Ind}(f, g ;(x, y))=1$; if it has the opposite orientation, we define $\operatorname{Ind}(f, g ;(x, y))=-1$. We then define

$$
\operatorname{Ind}(f, g ; J)=\sum_{(x, y) \in J} \operatorname{Ind}(f, g ;(x, y)) .
$$

Clearly, $\operatorname{Ind}(f, g ; J)=(-1)^{p q} \operatorname{Ind}(g, f ; J)$. Of course, to show that this is well defined, it must be shown that the quantity $\operatorname{Ind}(f, g ; J)$ is independent of the transverse approximations of $f$ and $g$ chosen.

On the other hand, $\operatorname{Ind}(f, g ; J)$ can be defined homologically. To do so, choose neighborhoods $J \subset U \subset V$ in $X \times Y$ such that $\bar{U} \subset V^{\circ}$ and $V \cap \operatorname{Int}(f, g)=J$. Then consider the composition

$H_{n}(X \times Y) \rightarrow H_{n}(X \times Y, X \times Y \backslash U) \stackrel{\cong}{\longleftarrow} H_{n}(V, V \backslash U) \stackrel{(f \times g)_{*}}{\longrightarrow} H_{n}(Z \times Z, Z \times Z \backslash \Delta(Z))$.

Since $X \times Y$ and $Z$ are compact orientable $n$-manifolds,

$$
H_{n}(X \times Y) \cong H_{n}(Z \times Z, Z \times Z \backslash \Delta(Z)) \cong \mathbb{Z},
$$

so the image of the generator of $H_{n}(X \times Y)$ under this composition gives an integer quantity $\operatorname{Ind}(f, g ; J)$. Of course, here the index must be shown to be independent of the neighborhoods $U$ and $V$ chosen.

Omitting a number of proofs (including the proof that the two definitions coincide), we have an integer index with the following properties:

1. Given $F: X \times[0,1] \rightarrow Z$ and $G: Y \rightarrow Z$, consider the "fat homotopies" $\mathbb{F}:$ $X \times[0,1] \rightarrow Z \times[0,1]$ and $\mathbb{G}: Y \times[0,1] \rightarrow Z \times[0,1]$, defined by $\mathbb{F}(x, t)=(F(x, t), t)$, $\mathbb{G}(y, t)=(G(y, t), t)$. If $\mathcal{J} \subset \operatorname{Int}(\mathbb{F}, \mathbb{G})$ is an isolated set of intersections, then so is every $J_{t_{0}}=\mathcal{J} \cap\left\{t=t_{0}\right\}$, and $\operatorname{Ind}\left(F_{t}, G_{t} ; J_{t}\right)$ is independent of $t$.

2. If $J=\emptyset$, then $\operatorname{Ind}(f, g ; J)=0$.

3. If $J_{1}, \ldots, J_{n}$ are disjoint isolated intersection sets, then

$$
\operatorname{Ind}\left(f, g ; \bigcup_{k=1}^{n} J_{k}\right)=\sum_{k=1}^{n} \operatorname{Ind}\left(f, g ; J_{k}\right) \text {. }
$$


4. Suppose $X=X_{1} \times X_{2}, Y=Y_{1} \times Y_{2}$, and $Z=Z_{1} \times Z_{2}$, with $\operatorname{dim}\left(X_{i}\right)+\operatorname{dim}\left(Y_{i}\right)=$ $\operatorname{dim}\left(Z_{i}\right)$. Given $f_{i}: X_{i} \rightarrow Z_{i}$ and $g_{i}: Y_{i} \rightarrow Z_{i}$ and isolated isolated intersection sets $J_{i} \subset \operatorname{Int}\left(f_{i}, g_{i}\right)$, define $f=f_{1} \times f_{2}, g=g_{1} \times g_{2}$ and $J=J_{1} \times J_{2}$. Then

$$
\operatorname{Ind}(f, g ; J)=\operatorname{Ind}\left(f_{1}, g_{1} ; J_{1}\right) \operatorname{Ind}\left(f_{2}, g_{2} ; J_{2}\right) .
$$

5. If $(x, y)$ is an isolated intersection of $f$ and $g$ with $\operatorname{Ind}(f, g ;(x, y))=0$ and $U \times V$ is a neighborhood of $(x, y)$ in $X \times Y$, then there are homotopies $F$ and $G$ such that $F_{0}=f$, and $G_{0}=g ; \operatorname{Int}\left(F_{1}, G_{1}\right)=\emptyset ;$ and $F_{t}=F_{0}$ and $G_{t}=G_{0}$ on $X \backslash U$ and $Y \backslash V$ respectively. Moreover, these homotopies can be chosen arbitrarily close to $f$ and $g$.

The index of the total intersection set $L I(f, g)=\operatorname{Ind}(f, g ; \operatorname{Int}(f, g))(\#(f, g)$ in $[7])$ is the (Lefschetz) intersection number of $f$ and $g$. It should be thought of as an analogue to the Lefschetz number for fixed points or coincidences. From the properties above, it is clear that

THEOREM 2.1. $L I(f, g)$ is a homotopy invariant, and if $L I(f, g) \neq 0$, then for every $f^{\prime} \simeq f, g^{\prime} \simeq g, \operatorname{Int}\left(f^{\prime}, g^{\prime}\right)$ is non-empty.

However, $L I(f, g)$ also suffers from the same limitations as the Lefschetz number: when non-zero, it does not estimate the number of intersections; and when zero, it does not guarantee that $f$ and $g$ are intersection-free.

Example 2.2. Consider the example from [7, Figure 5-3] shown in Figure 1. Let $X=Y=S^{1}, Z=T^{2} \# T^{2}$, and consider the submanifolds $M$ and $N$ to be the images of embeddings $f, g: S^{1} \rightarrow T^{2} \# T^{2}$. There are two intersections of opposite orientation, so $L I(f, g)=0$. That is, $L I(f, g)=0$ does not imply that $f$ and $g$ are intersection-free. Moreover, it appears that no deformation of $f$ or $g$ will remove the intersections, so $L I(f, g)=0$ does not even imply that $f$ and $g$ can be deformed to be intersection-free. This picture is, in some sense, a motivating example: neither intersection point can be removed individually by a deformation of $f$ or $g$; and the two intersection points cannot be "cancelled" because we cannot deform the image of $f$ into the image of $g$. The essense of the definition of the Nielsen intersection number lies in making these ideas precise and general.

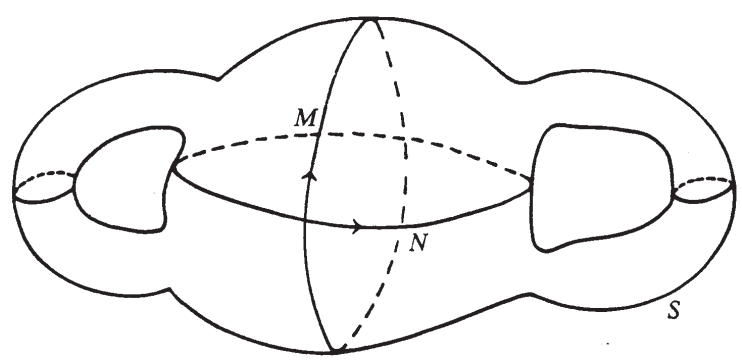

Fig. 1. Essential intersections in the double torus

If the manifolds are not all orientable, we can define a mod 2 index. That is, if we perturb $f$ and $g$ to be transverse, we count mod 2 the number of intersections. If we 
consider the homological approach, we use $\mathbb{Z}_{2}$ coefficients. In either case, we obtain a $\mathbb{Z}_{2}$-valued index which satisfies the same set of properties.

In fact, it is worth noting that we can, in principle, employ the homological definition of the intersection index in complete generality. The composition

$H_{n}(X \times Y) \rightarrow H_{n}(X \times Y, X \times Y \backslash U) \stackrel{\cong}{\longleftarrow} H_{n}(V, V \backslash U) \stackrel{(f \times g)}{\longrightarrow} H_{n}(Z \times Z, Z \times Z \backslash \Delta(Z))$ is still defined, and we can consider

$$
\operatorname{Int}(f, g ; J) \in \operatorname{Hom}\left(H_{n}(X \times Y), H_{n}(Z \times Z ; Z \times Z \backslash \Delta(Z))\right)
$$

to be the composition homomorphism. The first four properties enumerated remain valid, but the last one (removability of an isolated intersection with trivial index) does not. However, it is still true that the first two properties together imply that if $I(f, g ; J) \neq 0$, then $J$ cannot be deformed by a homotopy to the empty set.

Of course, the obvious problem with this is that, if either $\left.H_{n}(Z \times Z ; Z \times Z \backslash \Delta(Z))\right)$ or $H_{n}(X \times Y)$ is trivial, then all intersection classes automatically have index 0 . This is the shortcoming that prevents us from basing the theory in its full form on the intersection index. But, on the other hand, the index is useful (and computable) for more spaces than just manifolds.

3. Nielsen intersection numbers. As with any Nielsen-type number, the basic steps in the definition of the Nielsen intersection number are to partition the intersection set into intersection classes, define a notion of an essential class, and count the number of essential classes. The data required consists of compact, path connected spaces $X, Y$ and $Z$, and continuous functions $f: X \rightarrow Z$ and $g: Y \rightarrow Z$. We define an equivalence relation on $\operatorname{Int}(f, g)$ by $\left(x_{0}, y_{0}\right) \sim_{N}\left(x_{1}, y_{1}\right)$ if there exist paths $\alpha$ in $X$ from $x_{0}$ to $x_{1}$ and $\beta$ in $Y$ from $y_{0}$ to $y_{1}$ in $Y$ such that $f \alpha \simeq g \beta \operatorname{rel}\{0,1\}$. Alternatively, we can think of this as requiring a path $\omega$ in $X \times Y$ from $\left(x_{0}, y_{0}\right)$ to $\left(x_{1}, y_{1}\right)$ such that $(f \times g) \omega$ is homotopic rel $\{0,1\}$ to a path in the diagonal $\Delta(Z)$ in $Z \times Z$. Equivalence classes will be called intersection classes and the set of intersection classes will be denoted $\mathcal{I}(f, g)$.

Proposition 3.1. $\mathcal{I}(f, g)$ is finite, and each intersection class $J$ is a union of components of $\operatorname{Int}(f, g)$.

There is also a covering space approach to this partitioning of $I(f, g)$ into intersection classes. If $\tilde{X}, \tilde{Y}$ and $\tilde{Z}$ are the universal covers of $X, Y$ and $Z$, and $(x, y) \in \operatorname{Int}(f, g)$, choose $\tilde{x} \in p_{X}^{-1}(x)$ and $\tilde{y} \in p_{Y}^{-1}(y)$. Then there exists a covering transformation $\gamma \in \mathcal{D}(Z)$ such that $\tilde{f}(\tilde{x})=\gamma \tilde{g}(\tilde{y})$. Replacing $\tilde{g}$ by $\gamma \tilde{g}$, we can assume that $\tilde{f}(\tilde{x})=\tilde{g}(\tilde{y})$.

Proposition 3.2. For every $\gamma \in \mathcal{D}(Z),\left(p_{X} \times p_{Y}\right)(\operatorname{Int}(\tilde{f}, \gamma \tilde{g}))$ is an intersection class. Further, $\left(p_{X} \times p_{Y}\right)(\operatorname{Int}(\tilde{f}, \gamma \tilde{g}))=\left(p_{X} \times p_{Y}\right)\left(\operatorname{Int}\left(\tilde{f}, \gamma^{\prime} \tilde{g}\right)\right)$ if and only if there exist $\alpha \in$ $\mathcal{D}(X), \beta \in \mathcal{D}(Y)$ such that $\left(f_{\#} \alpha\right) \gamma=\gamma^{\prime}\left(g_{\#} \beta\right)$. Otherwise, $\left(p_{X} \times p_{Y}\right)(\operatorname{Int}(\tilde{f}, \gamma \tilde{g}))$ and $\left(p_{X} \times p_{Y}\right)\left(\operatorname{Int}\left(\tilde{f}, \gamma^{\prime} \tilde{g}\right)\right)$ are disjoint.

To complete the definition of the Nielsen intersection number, we need a definition of an essential intersection class. When an intersection index is defined, we say that an intersection class $J$ is algebraically essential if $\operatorname{Ind}(f, g ; J) \neq 0$. Since intersection indices are not defined in general, we need a more general notion of an essential class. 
Following Brooks [1], we can define in full generality a concept of a topologically essential interesection class, which explicitly captures the meaning of "essential" as "unable to be removed by a homotopy."

To do so, we return to the fat homotopies introduced in the previous section. There, they were used (in the setting of orientable manifolds of the appropriate dimensions) to describe the homotopy invariance of the intersection index. It followed then that intersection classes with non-zero intersection index cannot be removed by a homotopy. We now use the fat homotopy construction to describe directly an essential intersection class. Consider homotopies $F: X \times[0,1] \rightarrow Z$ and $G: Y \times[0,1] \rightarrow Z$. Define $\mathbb{F}: X \times[0,1] \rightarrow Z \times[0,1]$ and $\mathbb{G}: Y \times[0,1] \rightarrow Z \times[0,1]$ by $\mathbb{F}(x, t)=(F(x, t), t)$ and $\mathbb{G}(y, t)=(G(y, t), t)$. Then $\operatorname{Int}(\mathbb{F}, \mathbb{G})=\bigcup_{t \in[0,1]} \operatorname{Int}\left(F_{t}, G_{t}\right)$. Further, the partitioning of $\operatorname{Int}(\mathbb{F}, \mathbb{G})$ into intersection classes preserves this decomposition:

Lemma 3.3. Supppose $J$ is an intersection class in $\mathcal{I}(\mathbb{F}, \mathbb{G})$ Then, for every $t \in[0,1]$, $J_{t}=J \cap X \times Y \times\{t\}$ is an intersection class for $F_{t}$ and $G_{t}$.

We say that $J_{0}$ and $J_{1}$ are $(F, G)$-related, or more informally, that $J_{0}$ can be deformed to $J_{1}$. This defines an equivalence relation on $\bigcup_{f^{\prime} \in[f], g^{\prime} \in[g]} \mathcal{I}\left(f^{\prime}, g^{\prime}\right)$ by declaring $J_{0} \in$ $\mathcal{I}\left(f^{\prime}, g^{\prime}\right)$ and $J_{1} \in \mathcal{I}\left(f^{\prime \prime}, g^{\prime \prime}\right)$ to be related if there exist homotopies $F, G$ such that $J_{0}$ and $J_{1}$ are $(F, G)$-related. Now, given $J \in \mathcal{I}(f, g)$, we declare $J$ to be inessential if it can be deformed to the empty set. $J$ is essential if, for every $F: X \times[0,1] \rightarrow Z$ and $G: Y \times[0,1] \rightarrow Z$ with $F_{0}=f, G_{0}=g$, the set $\operatorname{Int}\left(F_{1}, G_{1}\right)$ is non-empty.

The Nielsen intersection number $N I(f, g)$ is the number of essential intersection classes in $\mathcal{I}(f, g)$. Clearly, from its construction, $N I(f, g) \leq|I(f, g)|$. Moreover, for every $f^{\prime} \simeq f, g^{\prime} \simeq g, N I(f, g)=N I\left(f^{\prime}, g^{\prime}\right)$. That is,

Theorem 3.4. $N I(f, g)$ is a homotopy invariant, and $N I(f, g) \leq M I_{c}(f, g)$.

ExAmple 3.5. Returning to the double-torus example of Example 2.2, it is clear that the two intersection points lie in different intersection classes. To see this, take the reprsentation

$$
\left\langle\alpha_{1}, \beta_{1}, \alpha_{2}, \beta_{2} \mid \alpha_{1} \beta_{1} \alpha_{1}^{-1} \beta_{1}^{-1}=\alpha_{2} \beta_{2} \alpha_{1}^{-1} \beta_{2}^{-1}\right\rangle
$$

of $\pi_{1}(S)$. Then $\pi_{1}(M)=\left\langle\alpha_{1} \beta_{1} \alpha_{1}^{-1} \beta_{1}^{-1}\right\rangle$ and $\pi_{1}(N)=\left\langle\beta_{1} \beta_{2}^{-1}\right\rangle$. Then, if we take any choice of paths $\alpha$ and $\beta$ in $S^{1}$ between the intersection points, then

$$
\left[f \alpha *(g \beta)^{-1}\right]=\left(\alpha_{1} \beta_{1} \alpha_{1}^{-1} \beta_{1}^{-1}\right)^{m} * \beta_{1}\left(\beta_{1} \beta_{2}^{-1}\right)^{n}
$$

for some choice of $m$ and $n$. No choice of $m$ and $n$ ever renders the trivial element (no choice of $m$ and $n$ will allow all of the $\alpha_{1}$ terms to cancel), so this loop is essential and the two intersection points are in different intersection classes. Since both classes consist of a single intersection point with index \pm 1 , both classes are essential, and

$$
N I_{a}(f, g)=N I(f, g)=M I_{c}(f, g)=M I_{p}(f, g)=2 .
$$

We will see in Theorem 5.1 that this is an example of a more general phenomenon.

EXAMPLE 3.6. Note that $N I(f, g)$ is not a homotopy-type invariant. For example, take $X=Y=Z=S^{1}$ and $f=g=i d$. Since the intersection set $\Delta\left(S^{1}\right) \subset S^{1} \times S^{1}$ is connected, there is only one intersection class. And, since any two maps $f^{\prime}, g^{\prime}$ homotopic 
to the identity must map onto $Z$, there is for every $z \in Z$ an $x \in X$ and $y \in Y$ such that $f^{\prime}(x)=g^{\prime}(y)=z$. In particular, $\operatorname{Int}\left(f^{\prime}, g^{\prime}\right) \neq \emptyset$, so the intersection class can never be removed by a homotopy. Thus there is one essential class, and $N I(i d, i d)=1$. But, if we replace $Z$ by $S^{1} \times[0,1]$, and define $f(x)=(x, 0), g(y)=(y, 1)$, then $\operatorname{Int}(f, g)=\emptyset$, and $N I(f, g)=0$.

4. The Wecken problem. Before we give the proof of Theorem 1.1, we need to consider more carefully some of the issues raised in the Introduction. Four quantities, $N I_{a}(f, g), N I(f, g), M I_{c}(f, g)$ and $M I_{p}(f, g)$ have been introduced. Are all four really necessary or appropriate? Other than the inequalities already noted, what can we say about the relationships between these quantities?

4.1. Comparing $M I_{c}$ and $M I_{p}$. We begin with the two "minimal" quantities $M I_{p}(f, g)$ and $M I_{c}(f, g)$. As the direct analogue of the quantity $M F(f)$ (the minimum number of fixed points in a homotopy class) considered in fixed point theory, $M I_{p}(f, g)$ is certainly the more familiar object. While counting the number of intersection points is a natural thing to do, it does have a disadvantage. For instance, suppose $X, Y$ and $Z$ are compact manifolds. The minimum $M I_{p}(f, g)$ will clearly be obtained when $f$ and $g$ intersect transversely. If $\operatorname{dim}(X)+\operatorname{dim}(Y)<\operatorname{dim}(Z)$, this means they do not intersect at all, and $M I(f, g)=0$. If $\operatorname{dim}(X)+\operatorname{dim}(Y)=\operatorname{dim}(Z)$, then the intersection set is discrete, and hence finite. But if $\operatorname{dim}(X)+\operatorname{dim}(Y)>\operatorname{dim}(Z)$, the intersection set will have positive dimension. To simply define $M I_{p}(f, g)=\infty$ in cases like this is to forgo useful information. By counting path components, the quantity $M I_{c}(f, g)$ avoids this shortcoming. If $X, Y$ and $Z$ are compact manifolds, this quantity will be finite without any conditions on $\operatorname{dim}(Z)-\operatorname{dim}(X)-\operatorname{dim}(Y)$. For this reason, it was $M I_{c}(f, g)$, rather than $M I_{p}(f, g)$, that was considered in [10]. ${ }^{2}$ The use of $M I_{c}(f, g)$ is not completely clear cut either. To see why, consider the following example.

EXAmple 4.1. Let $X=Y=S^{1}$, and let $Z$ consist of two copies $D^{+}, D^{-}$of the 2-disk $D^{2}$, with the identification $(1,0,+) \sim(1,0,-)$ and $(-1,0,+) \sim(-1,0,-)$. Then $Z \simeq S^{1}$, and any essential closed curve in $Z$ must pass through the two points $(1,0)$ and $(-1,0)$. Let $f, g: S^{1} \rightarrow Z$ be representatives of the generator of $\pi_{1}(Z)$. Then $f$ and $g$ must intersect in at least two points, and there are clearly choices of $f$ and $g$ which intersect only at those two points. Thus $M I_{p}(f, g)=2$. But if we choose $f=g$ to be an embedding, then $\operatorname{Int}(f, g)=\Delta\left(S^{1}\right)$ in $S^{1} \times S^{1}$, and $M I_{c}(f, g)=1$. Now, the functions $(f, g)$ with $M I_{p}(f, g)$ points of intersection cannot be the same as the functions $\left(f^{\prime}, g^{\prime}\right) \simeq(f, g)$ with $M I_{c}(f, g)$ components to the intersection set. Deforming $(f, g)$ to $\left(f^{\prime}, g^{\prime}\right)$ does reduce the number of path components of $\operatorname{Int}(f, g)$ from one to two, but it does so at the price of creating an arc of intersections.

The two local separating points in $Z$ make this example somewhat artificial, but the point it illustrates is a valid one: decreasing the number of components of $\operatorname{Int}(f, g)$ can increase the number of intersection points. If we are considering problems in which $X, Y$ and $Z$ compact manifolds with $\operatorname{dim}(X)+\operatorname{dim}(Y)=\operatorname{dim}(Z)$, then $\operatorname{Int}(f, g)$ will generically

\footnotetext{
${ }^{2}$ It is denoted there as $M I(f, g)$.
} 
be discrete, and it is really rather artificial to deform a finite set of intersections to an arc. That is, in this setting, $M I_{p}(f, g)$ really is the appropriate quantity to consider. Therefore, since each quantity has its appropriate role, we resist the temptation to declare one of the two as the minimum intersection number, and instead work with both.

The need to decide which quantity is the "right" one is diminished somewhat by Theorem 1.1, which asserts that $M I_{c}(f, g)$ and $M I_{p}(f, g)$ coincide for compact orientable manifolds (of high enough dimension) with $\operatorname{dim}(X)+\operatorname{dim}(Y)=\operatorname{dim}(Z)$. Theorems 5.1 and 5.3 will also show that $M I_{c}=M I_{p}$ for some maps into surfaces. However, it is still open whether this is true for low dimensional manifolds, or for non-orientable manifolds. The following low-dimensional, non-orientable "example" suggests that we can have $M I_{c}(f, g)<M I_{p}(f, g)$ in such situations.

ExAmple 4.2. Let $X=Y=S^{1}$, and let $M$ be a Möbius band. $M$ has universal cover $\tilde{M}=\mathbb{R} \times[-1,1]$, with covering transformations $(x, y) \cdot n=\left(x+n,(-1)^{n} y\right)$. We describe two maps $f, g: S^{1} \rightarrow M$ by defining functions $F, G:[0,1] \rightarrow \tilde{M}$ and extending them periodically. Let $F(t)=(3 t, 0)$, and let

$$
G(t)= \begin{cases}(5 t, 10 t-1) & 0 \leq t \leq \frac{1}{5} \\ (5 t, 1) & \frac{1}{5} \leq t \leq 1\end{cases}
$$

It is easy to see that $\operatorname{Int}(f, g)$ consists of three transverse intersection points. But, if we deform $G$ to $G^{\prime}$ with $G^{\prime}(t)=(5 t, 0)$, then $\operatorname{Int}(F, G) \subset S^{1} \times S^{1}$ is the curve $\left\{3 \theta_{1}=\right.$ $\left.5 \theta_{2}\right\}$, which is connected. Combining these two, we see that $N I_{a}(f, g)=N I(f, g)=$ $M I_{c}(f, g)=1$. Further, $M I_{p}(f, g)$ is either 1 or 3 . I conjecture that $M I_{p}(f, g)=3$, but at this writing, I have not been able to prove it. If so, this would provide an example, with manifolds with boundary, of $M I_{c}(f, g)<M I_{p}(f, g)$.

4.2. Comparing NI and $M I_{c}$. To establish that a pair of functions $f: X \rightarrow Z \leftarrow Y: g$ satisfies the weak Wecken property $N I(f, g)=M I_{c}(f, g)$, we must show that we can find an $f^{\prime} \simeq f$ and a $g^{\prime} \simeq g$ such that

- each essential class has been deformed to a path-connected set, and

- each inessential class has been removed.

Now, the information we are given almost guarantees that we can do this. First, the intuitive meaning of the Nielsen equivalence relation is that two intersection points lie in the same class if and only if they can be deformed into a single path component. Second, the definition of a topologically inessential class is one that can be removed via a homotopy. But, the definition does not guarantee that there is a single homotopy that removes all of the inessential classes simultaneously. Similarly, it is intuitively plausible that an essential class can be deformed into a single component, but this property does not follow instantly from the definition of an intersection class. It requires a proof, and that proof may well require hypotheses. That is, the equality $N I(f, g)=M I_{c}(f, g)$ may not be true in complete generality. On the other hand, at this point, I have no counterexamples - not even contrived ones.

Of course, Theorem 1.1 shows that the equality is true for manifolds of sufficiently high dimension. We will also see in Theorems 5.1 and 5.3 that the result is valid in some 
cases when the range space $Z$ is a surface. But in all of these theorems, it is the strong Wecken property that is established, and the proofs do not really provide any insight into how the weak Wecken property might be proven in greater generality.

4.3. Comparing $N I_{a}$ and $N I$. The last comparison, that between the two Nielsen numbers $N I(f, g)$ and $N I_{a}(f, g)$, turns out to be the most important in the strong Wecken property and the proof of Theorem 1.1. As discussed in $\S 2$, we can define an intersection index, and hence $N I_{a}$, in great generality. But it is only in restricted cases such as closed manifolds that the index is a consistently useful quantity. Since the semi-index for nonorientable manifolds has not yet been carried over to the intersection problem, we will confine our attention to compact orientable manifolds with $\operatorname{dim}(X)+\operatorname{dim}(Y)=\operatorname{dim}(Z)$.

In this setting, we can consider topologically essential classes (i.e. those that cannot be removed by a homotopy), or algebraically essential classes (i.e. those with non-zero index). Since a topologically inessential class has the index of the empty set, it is algebraically inessential. But is the converse true? That is, if $J$ is an intersection class with $\operatorname{Ind}(f, g ; J)=0$, can $J$ be deformed to the empty set? Again, the analogous results in the fixed point, coincidence and root problems suggest that this should be true if the dimensions of the manifolds are high enough, but may be false in low dimensional cases.

The first half of this analogy, at least, is valid. Algebraically inessential classes are topologically inessential if $\max \{\operatorname{dim}(X), \operatorname{dim}(Y)\}>2$. There are similar restrictions in the coincidence and root problems, and they all arise for the same reason. All of these results rest ultimately upon the Whitney trick [12], and must respect the restrictions present there:

Lemma 4.3 (Whitney Lemma). Consider embeddings $f: D^{p} \rightarrow M$ and $g: D^{q} \rightarrow M$, where $M$ is a compact orientable manifold of dimension $p+q$ and $p, q>2$. Suppose Int $(f, g)$ consists of exactly two points $\left(x_{1}, y_{1}\right),\left(x_{2}, y_{2}\right)$ with $\operatorname{Ind}\left(f, g ;\left(x_{1}, y_{1}\right)\right)=$ $-\operatorname{Ind}\left(f, g ;\left(x_{2}, y_{2}\right)\right)$. If there are paths $\alpha$ from $x_{1}$ to $x_{2}$ and $\beta$ from $y_{1}$ to $y_{2}$ such that $f \alpha$ is smoothly homotopic to $g \beta$, then there exists a smooth homotopy $F: D^{p} \times[0,1] \rightarrow M$ such that $F_{0}=f$ and $\operatorname{Int}\left(F_{1}, g\right)=\emptyset$.

Using this, we can show that

Lemma 4.4 ([10, Lemma 4.2]). Suppose $X, Y, Z$ are compact orientable manifolds with $\operatorname{dim}(X)+\operatorname{dim}(Y)=\operatorname{dim}(Z)$ and $\max \{\operatorname{dim}(X), \operatorname{dim}(Y)\}>2$. If $J \in \mathcal{I}(f, g)$ has $\operatorname{Ind}(f, g ; J)=0$, then there exist homotopies $F: X \times[0,1] \rightarrow Z$ and $G: Y \times[0,1] \rightarrow Z$ such that $J$ is F, G-related to the empty set.

Proof. Since $f$ and $g$ intersect transversely, the only way $J$ can have index 0 is for $J$ to consist of $2 m$ points, half of which have index +1 , the other half having index -1 . To remove $J$, it suffices to show that we can cancel a pair of points $(x, y),\left(x^{\prime}, y^{\prime}\right)$. If we assume without loss that $\operatorname{dim}(X) \leq \operatorname{dim}(Y)$, we must consider separately the two cases $\operatorname{dim}(X)<\operatorname{dim}(Y)$ and $\operatorname{dim}(X)=\operatorname{dim}(Y)$. In the former, $\operatorname{dim}(Z)>2 \operatorname{dim}(X)$, and an arbitrarily small perturbation will make $f$ an embedding [7, Theorem 2.2.13]. In the latter, $\operatorname{dim}(Z)=2 \operatorname{dim}(X)$, and an arbitrarily small perturbation will make $f$ an immersion [7, Theorem 2.2.12 \& Ex. 3.2.1] with clean double points. That is, there is a finite set $S_{f} \subset X$ such that 
1. $f$ is injective on $X \backslash S_{f}$.

2. For each $x \in S_{f}$ there is a neighborhood $U$ such that $\left.f\right|_{U}$ is injective.

3. If $f\left(x_{i}\right)=f\left(x_{j}\right)$, then $f\left(U_{i}\right) \cap f\left(U_{j}\right)=f\left(x_{i}\right)$, and $\left.f\right|_{U_{i}}$ and $\left.f\right|_{U_{j}}$ are transverse at that point.

Since $\operatorname{dim}(Z)=2 \operatorname{dim}(Y)$ as well, a similar deformation of $g$ can be made, and a similar set $S_{g}$ defined.

CASE I: $\operatorname{dim}(X)<\operatorname{dim}(Y)$. To apply the Whitney lemma, both of the maps involved must be embeddings. Since embeddings are not dense in $C^{0}(Y, Z)$, we must "manufacture" an embedding. To do so, consider $f \times i d: X \times Y \rightarrow Z \times Y$, and $\hat{g}: Y \rightarrow Z \times Y$ defined by $\hat{g}(y)=(g(y), y)$. These are both embeddings of manifolds with dimension at least 3. If $\delta: Y \rightarrow Y \times Y$ is the diagonal embedding, then $i d \times \delta: X \times Y \rightarrow X \times Y \times Y$ maps $\operatorname{Int}(f, g)$ homeomorphically to $\operatorname{Int}(f \times i d, \hat{g})$. There are paths $\alpha \times \beta$ from $(x, y)$ to $\left(x^{\prime}, y^{\prime}\right)$ in $X \times Y$ and $\beta$ from $y$ to $y^{\prime}$ in $Y$ such that $f \alpha \times \beta \simeq g \beta \times \beta$ in $Z \times Y$. To apply the Whitney lemma, we must verify that $\operatorname{Ind}(f \times i d, \hat{g} ;(x, y, y))=\operatorname{Ind}(f, g ;(x, y))$ and $\operatorname{Ind}\left(f \times i d, \hat{g} ;\left(x^{\prime}, y^{\prime}, y^{\prime}\right)\right)=\operatorname{Ind}\left(f, g ;\left(x^{\prime}, y^{\prime}\right)\right)$. See [10] for the details of this routine verification.

The Whitney lemma then implies that there exists a $\hat{G}: Y \times[0,1] \rightarrow Z \times Y$ such that $\hat{G}_{0}=\hat{g}$ and $\operatorname{im}\left(\hat{G}_{1}\right) \cap(f \times i d)=\emptyset$. Let $G$ be the projection $\rho_{1} \circ \hat{G}: Y \times[0,1] \rightarrow Z$ of $\hat{G}$ onto $Z$. It is a simple calculation to see that $f \times i d$ and $\hat{G}_{1}$ are intersection-free if and only if $f$ and $G_{1}$ are. This, then, is the required homotopy. Moreover, since the Whitney lemma is really a local result, $\hat{G}$ and $G$ can be constructed so that $g$ is deformed only in a neighborhood of $\beta$. In particular, no new intersection points are formed. Applying this process iteratively removes all of the intersection points in $J$.

CASE II: $\operatorname{dim}(X)=\operatorname{dim}(Y)$. If $\operatorname{dim}(Z)=2 \operatorname{dim}(X)=2 \operatorname{dim}(Y)$, then arbitrarily small perturbations make $f$ and $g$ immersions with clean double points and with transverse intersections. Moreover, since $f$ and $g$ each have only a finite number of self-intersections, we may assume that $\operatorname{Int}(f, g)$ is disjoint from $X \times S_{g}$ and $S_{f} \times Y$; and that if $\alpha$ and $\beta$ are paths connecting points in $J$, then the homotopy $H: f \alpha \simeq g \beta$ is likewise disjoint from $f\left(S_{f}\right)$ and $g\left(S_{g}\right)$. Now, take a neighborhood $V$ of $\alpha([0,1])$ which avoids $S_{f}$, and whose only intersections with $J$ are $x_{1}$ and $x_{2}$. The Whitney lemma implies that there is a homotopy $F: X \times[0,1] \rightarrow Z$ such that $F_{0}=f, F_{t}=f$ outside of $V$ for every t, and $F_{1}(V) \cap g(Y)=\emptyset$. Applying this process successively to pairs of points in $J$ eventually eliminates all intersections in $J$.

Thus we know that

Theorem 4.5. If $X, Y$ and $Z$ are compact orientable manifolds with $\operatorname{dim}(Z) \geq$ $\operatorname{dim}(X)+\operatorname{dim}(Y)$ and $\max \{\operatorname{dim}(X), \operatorname{dim}(Y)\}>2$, then for any $f: X \rightarrow Z$ and $g: Y \rightarrow Z$, an intersection class $J$ is topologically essential if and only if it is algebraically essential. That is, $N I_{a}(f, g)=N I(f, g)$.

5. Some Wecken theorems. We are now ready to present some results: first we give the proof of Theorem 1.1 for "high-dimensional" manifolds; then we present some results for surfaces. With one possible exception, these are instances of the strong Wecken 
property. The one possible exception arises if $Z=M$, the Möbius band. Theorem 5.1 will show that the weak Wecken property holds for the triple $\left(S^{1}, S^{1}, M\right)$, while Example 4.2 suggests that the strong form may not.

5.1. Proof of Theorem 1. The case $\operatorname{dim}(Z)>\operatorname{dim}(X)+\operatorname{dim}(Y)$ is trivial: in this case, transversality allows us to perturb $f$ and $g$ to be intersection free. The case we are really interested in is $\operatorname{dim}(Z)=\operatorname{dim}(X)+\operatorname{dim}(Y)$. As with Lemma 4.4, we assume $\operatorname{dim}(Y) \geq \operatorname{dim}(X)$. We follow the canonical construction to obtain $f^{\prime} \simeq f, g^{\prime} \simeq g$ such that $\left|\operatorname{Int}\left(f^{\prime}, g^{\prime}\right)\right|=N(f, g)$ :

1. If an intersection class $J$ has index 0 , deform $f$ and $g$ to remove $J$.

2. If an intersection class has non-zero index and contains more than one point, deform $f$ and $g$ to consolidate the class into a single point.

Lemma 4.4 takes care of the first step. For the second step, suppose $J$ is an intersection class with $\operatorname{Ind}(f, g ; J)=\nu \neq 0$. We may apply the same process to cancel all intersections in $J$ with indices of opposite sign, so we may assume that $J$ consists of $|\nu|$ intersection points, all having the same index. We may also assume that either $f$ is an embedding (if $\operatorname{dim}(X)<\operatorname{dim}(Y)$ ) or both $f$ and $g$ are immersions with clean double points (if $\operatorname{dim}(X)=\operatorname{dim}(Y))$. Choose a point $\left(x_{0}, y_{0}\right) \in J$, and euclidean neighborhoods $V$ of $y_{0}$ and $W$ of $g\left(y_{0}\right)$ such that $V \cap \pi_{2}(\operatorname{Int}(f, g))=y_{0} ; V \cap S_{g}=\emptyset$ and $W \cap\left(f\left(S_{f}\right) \cup g\left(S_{g}\right)\right)=\emptyset$ (if $S_{f}$ is non-empty); and $f(V) \subset W$. Now, $f(X) \cap W$ is a submanifold of $W$, so we are in the same setting as $[4, \S 3.3]$. The argument used there may be employed to produce points $y_{1}, \ldots, y_{|\nu|}, \bar{y} \in V$ and $g^{\prime} \simeq g$ such that

1. $g^{\prime}=g$ outside of $V$.

2. $\pi_{2}\left(\operatorname{Int}\left(f, g^{\prime}\right)\right) \cap V=\left\{y_{0}, y_{1}, \ldots, y_{|\nu|}, \bar{y}\right\}$.

3. All intersection points in $V$ lie in a single intersection class, which is related to $J$.

4. $\operatorname{Ind}\left(f, g^{\prime} ;\left(x_{0}, y_{0}\right)\right)=\operatorname{Ind}\left(f, g ;\left(x_{0}, y_{0}\right)\right), \operatorname{Ind}\left(f^{\prime}, g ;(\bar{x}, \bar{y})\right)=\nu$ and, for $i=1, \ldots,|\nu|$, $\operatorname{Ind}\left(f^{\prime}, g ;\left(x_{i}, y_{i}\right)\right)=-\operatorname{Ind}\left(f, g ;\left(x_{0}, y_{0}\right)\right)$.

Thus $J$ is deformed to an intersection class with $2|\nu|+1$ intersection points. Of these, $|\nu|$ have index $+1,|\nu|$ have index -1 and one has index $\nu$. Applying the arguments used in Lemma 4.4 again, we can cancel the $2|\nu|$ points with index \pm 1 , and continue $J$ to the single point $(\bar{x}, \bar{y})$ with index $\nu$.

It is worth noting in this result that, in all cases, we realized the lower bound $N I(f, g)$ by first deforming one map to an immersion with at most finitely many self-intersections, then deforming the other to eliminate all extra intersection points. We can interpret this as saying that the Nielsen intersection number is "almost" one-sided. That is, if we allow one arbitrarily small perturbation of one of the maps, we can eliminate all inessential classes by then holding that map fixed, and performing all deformations on the other map.

5.2. Superficial results. If we are considering closed orientable manifolds, we are left with three unresolved low-dimensional cases: $\operatorname{dim}(X)=\operatorname{dim}(Y)=1 ; \operatorname{dim}(X)=1$, $\operatorname{dim}(Y)=2$; and $\operatorname{dim}(X)=\operatorname{dim}(Y)=2$. Since the only compact 1-manifold is the circle, the case $\operatorname{dim}(X)=\operatorname{dim}(Y)=1$ is simply the case of two circles intersecting in 
a compact surface. But even in this simplest of cases, the situation is not completely clear. Example 4.2 suggests that the equality $M I_{c}(f, g)=M I_{p}(f, g)$ may not always hold. However, it is worth noting that, in that example, the surface was non-orientable, and the maps $f, g: S^{1} \rightarrow S$ could not be deformed to embeddings. We will see that, when $S$ is orientable and the maps are embeddings, we have equality. And there are two non-orientable examples, the Klein bottle and the projective plane, to show that orientability is not a necessary condition for equality.

THEOREM 5.1. Suppose $S$ is a closed surface, possibly with boundary, with non-negative Euler characteristic. Then, with one possible exception,

$$
|L I(f, g)| \leq N I_{a}(f, g)=N I(f, g)=M I_{c}(f, g)=M I_{p}(f, g)
$$

for any $f, g: S^{1} \rightarrow S$. The one possible exception is that the equality $M I_{c}(f, g)=$ $M I_{p}(f, g)$ may not hold if the surface is the Möbius band $M$ and $f_{\#}(1), g_{\#}(1) \in \pi_{1}(M)$ are both odd, and neither is a generator.

Proof. There are seven surfaces to be considered: the sphere $S^{2}$; the disk $D^{2}$; the projective plane $\mathbb{R P}^{2}$; the torus $T^{2}$; the cylinder $S^{1} \times \mathbb{I}$; the Klein bottle $K$ and the Möbius band $M$. If $S=S^{2}$ or $D^{2}$, then $S$ is simply connected, and every map $f: S^{1} \rightarrow S$ is inessential. Thus we can homotope $f$ and $g$ to non-intersecting constant maps. Similarly, Example 3.6 can be generalized to any pair of maps $f, g: S^{1} \rightarrow S^{1} \times \mathbb{I}$. That is, $f$ can be deformed into $S^{1} \times\{0\}$ and $g$ can be deformed into $S^{1} \times\{1\}$, so $\operatorname{Int}(f, g)=\emptyset$. For these three surfaces,

$$
|L I(f, g)|=N I_{a}(f, g)=N I(f, g)=M I_{c}(f, g)=M I_{p}(f, g)=0
$$

for all pairs of maps.

For $S=\mathbb{R P}^{2}, T^{2}, K$, we prove the Wecken theorem by choosing canonical representatives $f_{0}$ and $g_{0}$ for the homotopy classes $[f]$ and $[g]$, and showing that these representatives have exactly $N I(f, g)$ intersections. Suppose $p_{S}: \tilde{S} \rightarrow S$ is the universal cover of $S$, and $\tilde{f}_{0}, \tilde{g}_{0}: \mathbb{R} \rightarrow \tilde{S}$ are lifts of $f_{0}$ and $g_{0}$. We will show that, for every covering transformation $\gamma \in \mathcal{D}(S), p_{S} \operatorname{Int}\left(\tilde{f}_{0}, \gamma \tilde{g}_{0}\right)$ is either empty, or consists of a single transverse intersection of $f_{0}$ and $g_{0}$. This suffices, since a single transverse intersection must be essential.

If $S=\mathbb{R P}^{2}$, there is only one non-trivial homotopy class of maps. If either $f$ or $g$ is inessential, we can deform it to a constant map which does not intersect the image of the other map. The case when both are essential was presented as an example in [10]: Represent $\mathbb{R P}^{2}$ as a quotient space of the 2-disk $D^{2}$, with the antipodal identification on the boundary, and represent $f_{0}$ and $g_{0}$ by two distinct lines through the origin. Then $\mathcal{I}\left(f_{0}, g_{0}\right)$ consists of a single transverse intersection, so

$$
N I_{a}(f, g)=N I(f, g)=M I_{c}(f, g)=M I_{p}(f, g)=1 .
$$

For the torus and the Klein bottle, it will be more convenient to work on the universal covers. Both surfaces $T^{2}$ and $K$ have the plane as their universal cover. In either case, the maps $f_{0}, g_{0}: S^{1} \rightarrow S$ can be chosen so that their lifts $\tilde{f}_{0}, \tilde{g}_{0}: \mathbb{R} \rightarrow \mathbb{R}^{2}$ are straight lines through the origin. For both surfaces, the covering transformations $\mathcal{D}(S)$ are affine transformations, so $\gamma \tilde{g}_{0}$ is likewise a straight line. There are then three possibilities for $\operatorname{Int}\left(\tilde{f}_{0}, \gamma \tilde{g}_{0}\right)$ : the two lines do not intersect; they intersect in a single transverse 
point; or they coincide. If any $\operatorname{im}\left(\gamma \tilde{g}_{0}\right)$ coincides with $\operatorname{im}\left(\tilde{f}_{0}\right)$, then $\operatorname{im}\left(\tilde{g}_{0}\right)$ must coincide with $\operatorname{im}\left(\tilde{f}_{0}\right)$. By translating $\tilde{g}_{0}$ by an irrational amount in the direction perpendicular to $\operatorname{im}\left(\tilde{f}_{0}\right)$, we obtain a new $g_{0}$ such that every $\operatorname{Int}\left(\tilde{f}_{0}, \gamma \tilde{g}_{0}\right)$ is either empty, or consists of one transverse intersection point.

Finally, we consider the Möbius band. Example 4.2 was left unresolved because we can (at this point) neither prove nor disprove the equality $M I_{c}(f, g)=M I_{p}(f, g)$ for all maps into the Möbius band. However, the approach used for the torus and Klein bottle can be applied to prove $N I_{a}(f, g)=N I(f, g)=M I_{c}(f, g)$ for all maps $f, g: S^{1} \rightarrow M$. The Möbius band has universal cover $\mathbb{R} \times[-1,1]$. If either $f$ or $g$ wraps $S^{1}$ around $M$ an even number of times (WLOG, suppose $f$ does), then $f_{0}$ can be chosen so that $\operatorname{im}(f)=\partial M$. $g_{0}$ can be chosen so that $\operatorname{im}(g)$ is the "center circle" of $M$ (i.e. $\tilde{g}$ maps to $\mathbb{R} \times\{0\}$ ). Clearly, $\operatorname{Int}\left(f_{0}, g_{0}\right)=\emptyset$ in this case. On the other hand, suppose both $f$ and $g$ wrap around $M$ an odd number of times. If $f_{\#}(1)=2 n+1$ and $g_{\#}(1)=2 m+1$, we define representatives $f_{0}$ and $g_{0}$ by defining the lifts $\tilde{f}_{0}, \tilde{g}_{0}$. As before, it suffices to define $\left.\tilde{f}_{0}\right|_{[0,1]},\left.\tilde{g}_{0}\right|_{[0,1]}$ and extend periodically. These are defined as follows:

$$
\begin{aligned}
& \tilde{f}_{0}(t)=((2 n+1) t, 0), \\
& \tilde{g}_{0}(t)= \begin{cases}((2 m+1) t, 2(2 m+1) t-1), & 0 \leq t \leq \frac{1}{2 m+1}, \\
((2 m+1) t, 1), & \frac{1}{2 m+1} \leq t \leq 1 .\end{cases}
\end{aligned}
$$

Then $\operatorname{Int}\left(\tilde{f}_{0}, \tilde{g}_{0}\right)=\left\{\left(\frac{2 k+1}{2(2 n+1)}, \frac{1}{2(2 m+1)}\right) \mid 0 \leq k \leq 2 n\right\}$, so the Nielsen class $p \operatorname{Int}\left(\tilde{f}_{0}, \tilde{g}_{0}\right)$ consists of $2 n+1$ transverse intersection points. The mod 2 intersection number is nonzero, so the class is essential. But, if $\tilde{g}_{0}$ is deformed to $\tilde{g}_{0}^{\prime}(t)=((2 m+1) t, 0)$, then $\operatorname{Int}\left(\tilde{f}_{0}, \tilde{g}_{0}^{\prime}\right) \cong \mathbb{R}$ is connected, and $p_{M} \operatorname{Int}\left(\tilde{f}_{0}, \tilde{g}_{0}^{\prime}\right)$ is a connected essential intersection class.

We range over all intersection classes as we range over $p_{M} \operatorname{Int}\left(n \cdot \tilde{f}_{0}, \tilde{g}_{0}^{\prime}\right)$ for $n \in \mathcal{D}(M) \cong$ $\mathbb{Z}$. But, since the center line is left invariant by all covering transformations, every $n \cdot \tilde{f}_{0}$ is simply an integer translation of $\tilde{f}_{0}$, and every $p_{M} \operatorname{Int}\left(\tilde{f}_{0}, \tilde{g}_{0}^{\prime}\right)$ is likewise a connected essential intersection class. Thus $N I_{a}\left(f_{0}, g_{0}\right)=N I\left(f_{0}, g_{0}\right)=M I_{c}\left(f_{0}, g_{0}\right)$.

For the surfaces with positive Euler characteristic, we see that $N I(f, g)$ is never greater than one. But for the surfaces with Euler characteristic zero, we can see arbitrarily large values for $N I(f, g)$. To see this, we begin with the torus. The homotopy classes of the maps $f, g: S^{1} \rightarrow T^{2}$ are determined by the homomorphisms $f_{*}, g_{*}: H_{1}\left(S^{1}\right) \rightarrow H_{1}\left(T^{2}\right)$. Let $A=\left[f_{1 *} g_{1 *}\right]$ be the $2 \times 2$ integer matrix whose columns are the homomorphisms $f_{*}$ and $g_{*}$.

Theorem 5.2. For any $f, g: S^{1} \rightarrow T^{2}$, if $A=\left[f_{1 *} g_{1 *}\right]$, then

$$
|L I(f, g)|=N I(f, g)=|\operatorname{det}(A)| .
$$

Proof. If $\operatorname{det}(A)=0$, then $f_{0}$ and $g_{0}$ map into the same one-dimensional subspace of $T^{2}$. Translating $g_{0}$ an irrational amount in the perpendicular direction removes all intersections, so $L I(f, g)=N I(f, g)=0$. If $\operatorname{det}(A) \neq 0$, then $f_{0}$ and $g_{0}$ intersect transversally, and the argument above shows that every intersection class consists of a single transverse intersection. Moreover, since any intersection class has the form $p \operatorname{Int}\left(\tilde{f}_{0}, \gamma \tilde{g}_{0}\right)$, with the covering transformation $\gamma \in \mathcal{D}\left(T^{2}\right)$ a translation, all intersection points have the same index. This establishes that $|L I(f, g)|=N I(f, g)=\left|\operatorname{Int}\left(f_{0}, g_{0}\right)\right|$. It remains only to show 
that $\left|\operatorname{Int}\left(f_{0}, g_{0}\right)\right|=|\operatorname{det}(A)|$. Since $f_{0}$ and $g_{0}$ are homomorphisms, $\operatorname{Int}\left(f_{0}, g_{0}\right)=\operatorname{ker}(\Phi)$, where $\Phi: S^{1} \times S^{1} \rightarrow T^{2}$ is defined by $\Phi\left(\theta_{1}, \theta_{2}\right)=f_{0}\left(\theta_{1}\right)-g_{0}\left(\theta_{2}\right)$. The calculation that $|\operatorname{ker}(\Phi)|=|\operatorname{det}(A)|$ is a routine one.

In the special case of $f$ and $g$ both embedddings, this result is found in [6]; and it is in turn a special case of a formula in [11] for tori of all dimensions.

Next, we consider the Klein bottle $K$. Of course, $K$ is doubly covered by the torus. Given $f, g: S^{1} \rightarrow K$, there are covers $p_{1}, p_{2}: S^{1} \rightarrow S^{1}$ and lifts $\tilde{f}, \tilde{g}: S^{1} \rightarrow T^{2}$ such that the diagram commutes:

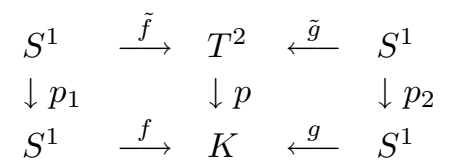

Let $\gamma: T^{2} \rightarrow T^{2}$ be the covering transformation over $K$, and let $d_{i}$ be the ordering of the cover $p_{i}$. It was shown in [10] that

$$
N I(f, g) \geq \frac{1}{d_{1} d_{2}}(N I(\tilde{f}, \tilde{g})+N I(\tilde{f}, \gamma \tilde{g}))
$$

This inequality need not be sharp, but it suffices for our purposes: since $N I(\tilde{f}, \tilde{g})$ can be arbitrarily large, and $d_{1}, d_{2} \leq 2$, it follows that $N I(f, g)$ can be arbitrarily large.

For the Möbius band, we saw that $N I(f, g)$ is non-zero only if $f$ and $g$ wrap around the band an odd number of times. The proof of Theorem 5.1 showed that, if we represent $f$ and $g$ by homomorphisms from $S^{1}$ to the "center circle" $S^{1}$, then every intersection class is connected and essential. So it suffices to compute the number of components of $\operatorname{Int}(f, g)=\operatorname{ker}(\Phi)$, where $\Phi$ here denotes the map $S^{1} \times S^{1} \rightarrow S^{1}$ given by $\Phi\left(\theta_{1}, \theta_{2}\right)=$ $f\left(\theta_{1}\right)-g\left(\theta_{2}\right)$. If $f$ is multiplication by $p$, and $g$ is multiplication by $q$, then it is a standard calculation that $\operatorname{ker}(f-g)$ has $\operatorname{gcd}(p, q)$ components.

This, with one possible gap, shows that the intersection theory for surfaces with nonnegative Euler characteristic is well behaved. This is hardly surprising. We would expect any Nielsen theory for such surfaces to be quite simple, and it is somewhat surprising to find that there are any questions that cannot be answered in a routine fashion. For surfaces with negative Euler characteristic, on the other hand, we will not be surprised to encounter difficulties. If we again look to fixed point theory for analogies to help guide our thinking, we might conjecture that the Wecken property holds for embeddings of $S^{1}$, but not for arbitrary maps. At this writing, I have no examples of pairs of maps from $S^{1}$ to a surface of negative Euler characteristic for which any of the equalities

$$
N I_{a}(f, g)=N I(f, g)=M I_{c}(f, g)=M I_{p}(f, g)
$$

can be proven to be false.

However, the other half of the conjecture can be confirmed. When considering embeddings of $S^{1}$ into a surface of negative Euler characteristic, we have the very powerful techniques of Nielsen-Thurston theory to draw on [6]. There, one of the quantities of interest is $i(\alpha, \beta)$, the minimum number of intersections in an isotopy class of embeddings $\alpha$ and $\beta$. Two embeddings are homotopic if and only if they are isotopic, so

$$
i(\alpha, \beta)=\min \left\{\left|\operatorname{Int}\left(\alpha^{\prime}, \beta^{\prime}\right)\right| \mid \alpha^{\prime} \simeq \alpha, \beta^{\prime} \simeq \beta, \alpha^{\prime} \text { and } \beta^{\prime} \text { embeddings }\right\} .
$$


Clearly, if the homotopy classes $[\alpha]$ and $[\beta]$ contain embeddings, $N I_{p}(\alpha, \beta) \leq i(\alpha, \beta)$. From this observation, and the established properties of $i(\alpha, \beta)$, we deduce the following:

THEOREM 5.3. If $S$ is a closed orientable surface with $\chi(S)<1$, and $\alpha, \beta: S^{1} \rightarrow S$ are embeddings, then

$$
|L I(\alpha, \beta)| \leq N I_{a}(\alpha, \beta)=N I(\alpha, \beta)=M I_{c}(\alpha, \beta)=M I_{p}(\alpha, \beta)=i(\alpha, \beta)
$$

Proof. First note that if $\alpha \simeq \beta$, then we can take a tubular neighborhood $T$ around $\operatorname{im}(\alpha)$, and deform $\beta$ first into $\operatorname{im}(\alpha)$, and then into $T \backslash \operatorname{im}(\alpha)$. Then $\operatorname{Int}(\alpha, \beta)=\emptyset$. If $\alpha \not \beta$, it suffices to show that all lifts $\tilde{\alpha}$ and $\tilde{\beta}$ have the property that $\operatorname{Int}(\tilde{\alpha}, \tilde{\beta})$ is either empty or consists of a single transverse intersection. But [6, Exposé 3, Proposition 10] implies that every pair of distinct isotopy classes of simple closed curves in $S$ contains a pair of embeddings $(\alpha, \beta)$ with this property.

6. Concluding remarks. This investigation of the Wecken problem for intersection theory is only a beginning. There remain many open questions. In the most richly structured setting of closed orientable manifolds, we still have the low-dimensional gap. The surface case is not yet completely understood, and there is almost nothing know about the cases $\operatorname{dim}(X)=1, \operatorname{dim}(Y)=2$ and $\operatorname{dim}(X)=\operatorname{dim}(Y)=2$.

Here, the failure of the Whitney trick is likely to have significant consequences. In the case $\operatorname{dim}(X)=\operatorname{dim}(Y)=2$ in particular, it is very likely that there are examples for which the strong Wecken property fails. But this points to another gap in the theory: there are not yet any non-trivial examples of pairs of maps for which the strong Wecken property fails. The one example presented, Example 4.1, had local separting points, which really trivializes the problem of computing $M I_{p}(f, g)$. It is the difficulty of computing $M I_{p}(f, g)$ that stands as an obstacle to producing more meaningful counter-examples. Of course, it is this same difficulty that provides Nielsen theory's raison d'être: if quantities such as $M I_{p}(f, g)$ were easy to compute, there would be no need for Nielsen theory.

Throughout this paper, analogies to the Nielsen theories for fixed points, coincidences and roots have been drawn. We conclude with one more: the distinctions between $M I_{c}$ and $M I_{p}$, and between $N I_{a}$ and $N I$, are equally valid in these other problems. The Nielsen fixed point number is analoguous to $N I_{a}$, while the coincidence and root indices are analoguous to $N I$. In all of these problems, the "minimum quantity" considered is always of the form $M I_{p}$, and the Wecken theorems proved are always the strong form of the Wecken theorem. Jiang's non-Wecken example [9], for example is an example in which the strong Wecken property fails. But the weak Wecken property does hold there. That is, if $N_{a}(f)$ denotes the number of algebraically essential fixed point classes (i.e. the Nielsen fixed point number), $N_{t}(f)$ denotes the number of topologically essential fixed point classes (i.e. those that cannot be removed by a homotopy), and $M F_{c}(f)$ and $M F_{p}(f)$ have the obvious meanings, then what Jiang gave in [9] was an example in which $N_{a}(f)=0, N_{t}(f)=M F_{c}(f)=1$ and $M F_{p}(f)=2$.

There is an obvious motivational value to drawing analogies like this between the various problems. But the connection between these various Nielsen theories is stronger than that. In [4], Dobreńko and Kucharski point out that coincidence theory can be 
formulated as a special case of root theory. In fact, if we consider the three problems of coincidences, intersections and roots, it is possible to formally translate any one of the three problems into either of the others. It is then reasonable to ask how the various Nielsen theory quantities transform. This question will be considered in detail in [11].

These transformations have an interesting connection with the study of Wecken properties. Very briefly, if we start with the intersection problem $f: X \rightarrow Z \leftarrow Y: g$ with $X, Y$ and $Z$ closed orientable manifolds, then we can produce a coincidence problem $F, G: X \times Y \rightarrow Z$ with $F(x, y)=f(x)$ and $G(x, y)=g(y)$. This can in turn be transformed into the root problem $f \times g: X \times Y \rightarrow Z \times Z \supset \Delta(Z)$. These transformations have the properties:

$$
\begin{aligned}
M I_{p}(f, g) \geq M C_{p}(F, G) & =M R_{p}(f \times g ; \Delta(Z)) \\
M I_{c}(f, g) \geq M C_{c}(F, G) & =M R_{c}(f \times g ; \Delta(Z)) \\
N I(f, g) \geq N(F, G) & =N(f \times g ; \Delta(Z)) \\
N I_{a}(f, g) & =N_{a}(F, G)=N_{a}(f \times g: \Delta(Z))
\end{aligned}
$$

(where $M C_{p}$ and $M R_{p}$ have the obvious meanings: the minimum number of coincidence or roots in the homotopy class). The obvious question, and the central issue in [11], is whether these transformations (and the transformation from roots to intersections) is "Nielsen-number preserving." That is, is $N I(f, g)=N(F, G)$ for all $f$ and $g$ ?

Now, in all three theories, there is a strong Wecken theorem for closed orientable manifolds. From the inequalities above, it is clear that, if the strong Wecken theorem holds for a problem and for the transform of the problem, then the transformation must be Nielsen-number preserving. However, all three theorems rely on the Whitney trick, and so must exclude some low-dimensional cases. And, because they invoke the Whitney trick in slightly different ways, these low-dimensional exclusions are not perfectly preserved by the transformations. So, for example, if $\operatorname{dim}(X)=1$ or 2 and $\operatorname{dim}(Y)=2$, then $X, Y$ and $Z$ may not satisfy the strong Wecken property for intersections. But their transform into roots, $f \times g: X \times Y \rightarrow Z \times Z \supset \Delta(Z)$, does satisfy the hypotheses of [4, Theorem 3.4], and so does satisfy the strong Wecken property. The consequence of this is that, in this setting, a pair of maps $f$ and $g$ do satisfy the strong Wecken property for intersections if and only if their transformation into the root problem is Nielsen-number preserving.

\section{References}

[1] R. Brooks and R. F. Brown, A lower bound for the $\Delta$-Nielsen number, Trans. Amer. Math. Soc. 143 (1969), 555-564.

[2] R. Brooks, The number of roots of $f(x)=a$, Bull. Amer. Math. Soc. 76 (1970), 10501052 .

[3] R. Brooks, On the sharpness of the $\Delta_{2}$ and $\Delta_{1}$ Nielsen numbers, J. Reine Angew. Math. 259 (1973), 101-108.

[4] R. Dobreńko and Z. Kucharski, On the generalization of the Nielsen number, Fund. Math. 134 (1990), 1-14.

[5] R. Dobreńko and J. Jezierski, The coincidence Nielsen theory on non-orientable manifolds, Rocky Mountain J. Math. 23 (1993), 67-85. 
[6] A. Fathi, F. Laudenbach et V. Poénaru, Travaux de Thurston sur les surfaces, Séminaire Orsay, Astérisque 66-67 (1979).

[7] M. Hirsch, Differential Topology, Springer-Verlag, Berlin, 1976.

[8] B. Jiang, Lectures on Nielsen Fixed Point Theory, Contemp. Math. 14, Amer. Math. Soc., Providence, RI, 1983.

[9] B. JiAng, Fixed points and braids, Invent. Math. 75 (1984), 69-74.

[10] C. McCord, A Nielsen theory for intersection numbers, Fund. Math. 152 (1997), 117-150.

[11] C. MCCORD, The three faces of Nielsen: comparing coincidence numbers, intersection numbers and root numbers, in preparation.

[12] J. Milnor, Lectures on the h-Cobordism Theorem, Princeton Univ. Press, 1965. 